\title{
贵金属/半导体光电阳极在直接甲醇燃料电池中的应用
}

\author{
翟春阳 ${ }^{1}$, 孙明娟 ${ }^{1}$, 杜玉扣 ${ }^{2}$, 朱明山 ${ }^{1}$ \\ (1. 宁波大学 材料科学与化学工程学院, 宁波 315211; 2. 苏州大学 材料科学与化学化工学部, 苏州 215123)
}

摘 要: 直接甲醇燃料电池(DMFCs) 由于具有能量效率高, 携带方便和环境友好等特点, 作为新型清洁能源受到越 来越多的关注。阳极催化剂的优劣是影响 DMFCs 性能的关键因素之一。近年来研究显示, 利用具有光催化活性的 半导体材料作为贵金属催化剂的载体，在外界光源的照射下，能够极大地改善电极的电催化活性和稳定性。本文对 该类新型光响应贵金属/半导体电极在光照条件下对增强甲醇的电催化氧化性能方面进行了总结和概述。首先，阐 述了光照增强电极电催化甲醇氧化性能的基本反应机制; 然后, 对该类电极的制备方法以及催化活性等方面的研 究进展进行了系统总结; 最后，对该类电极在未来 DMFCs 中应用存在的问题和发展前景做了总结和展望。

关 键 词: 直接甲醇燃料电池; 半导体纳米材料; 电催化氧化; 光电阳极; 综述

中图分类号: TQ152 文献标识码: A

\section{Noble Metal/Semiconductor Photoactivated Electrodes for Direct Methanol Fuel Cell}

\begin{abstract}
ZHAI Chun-Yang ${ }^{1}$, SUN Ming-Juan ${ }^{1}$, DU Yu-Kou ${ }^{2}$, ZHU Ming-Shan ${ }^{1}$
(1. School of Materials Science and Chemical Engineering, Ningbo University, Ningbo 315211, China; 2. College of Chemistry, Chemical Engineering and Materials Science, Soochow University, Suzhou 215123, China)

Abstract: Direct methanol fuel cells (DMFCs) have received great attentions as clean energy due to their higher energy efficiency, easy transportation and environmental friendly. The activity and stability of the anode catalysts are the key factors of DMFCs. Recent studies show that usage of semiconductor materials as a supporter of traditional noble metal electrocatalyst greatly improves the electrocatalytic activity and stability of the electrode in DMFCs under light irradiation. In this paper, the research progress of photoactivated noble metal/semiconductor electrocatalyst for DMFCs in recent years is summarized including: (i) the mechanism of improving electrocatalytic performance of the noble metal/semiconductor electrode in DMFCs under light irradiation; (ii) the fabrication methods of noble metal/semiconductor electrode; and (iii) catalytic performance of the noble metal/semiconductor electrode. And the prospects and problems of the photo-responsive electrodes for DMFCs reactions are addressed briefly.
\end{abstract}

Key words: direct methanol fuel cells; semiconductor nanomaterials; electrocatalytic oxidation; photoelectrochemical anode; review

能源危机和环境污染已成为人类社会面临的严 重挑战 ${ }^{[1-6]}$ 。直接醇类然料电池(DAFC) 是一种可直接 利用醇类溶液作燃料的低温燃料电池, 由于其高效
率和低排放的特性 ${ }^{[1-13]}$, 已作为能量转换器被广泛研 究。甲醇和乙醇等由于具有相对高的质量能量密度、 易储存和运输等特点 ${ }^{[1-7]}$, 常作为液体燃料应用于 
DAFC。其中, 甲醇是能量密度最高的燃料 ${ }^{[8]}$, 且以甲 醇作为燃料, 无需中间重整或转化装置, 同时甲醇 具有来源丰富、价格低廉、易携带和储存等优点, 使 直接甲醇燃料电池(direct methanol fuel cells, DMFCs) 成为研究最广、发展最成熟的低温燃料电池 ${ }^{[4,11]}$ 。

催化剂材料是 DMFCs 的核心部件之一, 也是制 备高性能、低成本燃料电池的主要技术屏障。催化 剂主要由贵金属 ${ }^{[3]}$ 和载体 ${ }^{[2,12]}$ 两部分构成。铂 $(\mathrm{Pt})$ 是当 前使用最广泛的贵金属催化剂, 但其成本较高和抗 一氧化碳(CO)毒化能力较差 ${ }^{[14]}$, 限制了其在 DMFCs 中的应用。近年来, 科研人员一直致力于探索高效率 和低成本的催化剂材料 ${ }^{[3-14]}:$ (1)通过控制和改变贵金 属的尺寸、形貌以及引入其他金属等手段提高催化 剂的利用率; (2)探究新型贵金属载体, 利用载体降低 贵金属的负载量, 并改善催化剂催化活性和稳定性。 目前常用的催化剂载体材料有各种碳材料、导电高 分子材料和一些具有特殊结构的复合材料 ${ }^{[3,8-13]}$ 。

由于具有大的比表面积、优异的光学性质、化 学稳定性、热稳定性和共催化特性, 二氧化钛 $\left(\mathrm{TiO}_{2}\right)$ 和三氧化铇 $\left(\mathrm{WO}_{3}\right)$ 等半导体材料作为贵金属载体被 应用到 $\mathrm{DMFCs}^{[12,13,15-17]}$ 。半导体纳米材料的比表面 积大, 有利于贵金属催化剂的分散, 提高催化剂的 利用率, 从而提高催化剂的催化活性。半导体纳米 材料的稳定性优异, 有利于复合催化剂在 DMFCs 的强酸/强碱体系中保持稳定的状态。最重要的是, 传统半导体优异的光学性质使其在光激发下具有较 强的氧化能力, 能够氧化吸附在其表面的有机小分 子 $^{[18-20]}$, 从而进一步提高催化剂的催化活性和抗毒 化能力。尽管半导体材料在导电性方面与传统贵金 属或者碳载体相比还有待提高, 但是通过掺杂、改 变半导体形貌等手段, 半导体材料的导电性能够得 到明显的改善 ${ }^{[13,15]}$ 。例如 2005 年 Kamat 课题组 ${ }^{[21]}$ 制备了 $\mathrm{Pt}-\mathrm{Ru} / \mathrm{TiO}_{2}$ 复合电极, 并首次发现紫外光照 射下该电极对甲醇氧化的电流密度与无光照相比提 高了 $25 \%$ 。目前, 国内外研究者围绕贵金属/半导体 复合电极在光照射提高甲醇催化氧化性能中开展了 大量工作 ${ }^{[22-29]}$ 。在该体系中, 甲醇分子在光催化和 电催化的协同作用下被催化氧化。同时, 半导体材 料在光照下产生的具有强氧化性的空穴使得复合电 极具有明显的自清洁能力。基于此, 本文主要对当 前具有光响应的贵金属/半导体复合电极催化氧化 甲醇的机理、电极的制备方法以及不同类型半导体 载体在甲醇催化氧化中的应用等方面进行总结和概 述, 并对该类光响应电极未来发展的趋势和方向进 行总结和展望。

\section{1 光响应贵金属/半导体光电极的甲 醇催化氧化机理}

光照射下贵金属/半导体复合电极在催化甲醇的 过程中存在光催化和电催化的协同作用。以硫化镉 $(\mathrm{CdS})$ 负载贵金属 $\mathrm{Pt}$ 光电催化氧化甲醇为例 ${ }^{[30]}$ (如图 1 所示)。首先, 在电催化过程中, 甲醇先吸附在电极 表面, 经多步脱氢形成含碳中间毒化物种, 然后, 解 离水产生含氧物种，与含碳中间毒化物种发生反应， 最终氧化生成 $\mathrm{CO}_{2}$ 。另一方面, $\mathrm{CdS}$ 在光激发下生成 电子-空穴对 ${ }^{[18-20]}$, 空穴能够与吸附在 $\mathrm{CdS}$ 表面的 $\mathrm{OH}^{-} / \mathrm{H}_{2} \mathrm{O}$ 反应生成具有强氧化性的 $\cdot \mathrm{OH}^{[24-27]}, \cdot \mathrm{OH}$ 进一步氧化吸附在电极表面的 $\mathrm{CH}_{3} \mathrm{OH}$, 因此, 光照 射有助于提高催化剂对甲醇的催化氧化性能 ${ }^{[24-27]}$ 。 此外, $\cdot \mathrm{OH}$ 还可以氧化催化过程中生成的 $\mathrm{CO}$ 等中间 产物，从而有效抑制催化剂的 CO 中毒 ${ }^{[29,31]}$ 。同时, 在外加电压作用下, 光生电子移至外电路, 有效减 少了光生电子-空穴对的复合, 提高催化剂的催化 效率。因此, 光照射能够有效增强贵金属/半导体电 极对甲醇等有机小分子的催化活性、稳定性以及抗 毒化能力。

\section{2 贵金属/半导体电极的制备方法}

目前，贵金属/半导体复合电极常见的制备方法 主要有电化学沉积法、化学还原法、物理混合法、 水热/溶剂热法和微波辅助法等。

\section{1 电化学沉积法}

在现有的合成方法中, 电化学沉积法是最简单 且广受欢迎的方法, 这主要是因为电化学过程不仅 简单、快速且绿色, 更重要的是, 通过调节各种电沉

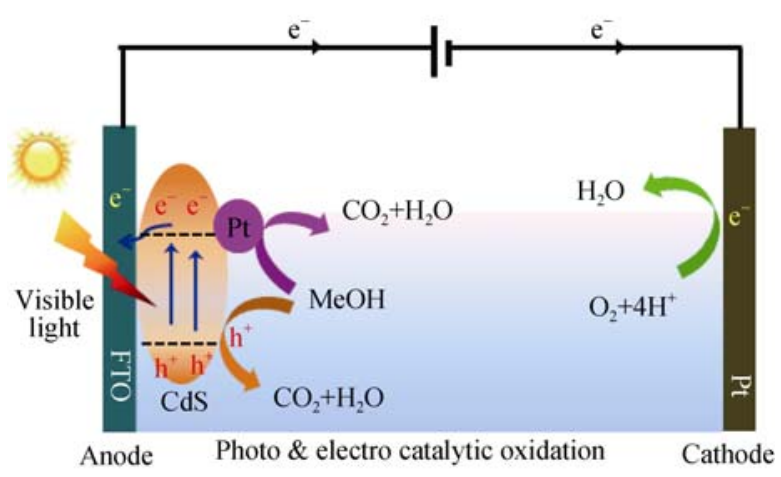

图 1 光照条件下 $\mathrm{Pt} / \mathrm{CdS}$ 电极光电催化甲醇的过程示意图 ${ }^{[30]}$ Fig. 1 Schematic illustration for photoelectrocatalytic methanol oxidation via $\mathrm{Pt} / \mathrm{CdS}$ electrode under light illumination ${ }^{[30]}$ 
积参数可以有效控制贵金属纳米结构的成核和生长 速率 ${ }^{[11]}$ 。例如 $\mathrm{He}$ 等 ${ }^{[32]}$ 以 $\mathrm{H}_{2} \mathrm{SO}_{4}+\mathrm{H}_{2} \mathrm{PtCl}_{4}$ 的混合溶 液为电解质, 采用恒电位脉冲电沉积法把直径约 $100 \mathrm{~nm}$ 的 $\mathrm{Pt}$ 纳米粒子均匀负载在 $\mathrm{TiO}_{2}$ 薄膜表面。 随后又用类似的方法将 $\mathrm{Pt}$ 纳米粒子沉积在 $\mathrm{TiO}_{2}-\mathrm{SiO}_{2}$ 表面 ${ }^{[33]}$ 。Zhang 等 ${ }^{[34]}$ 采用脉冲电沉积法合成了 $\mathrm{PtNi}$ 纳米粒子负载的 $\mathrm{TiO}_{2}$ 纳米管阵列(TNTs)阳极, 其中 直径约 50 80 nm 的 PtNi 纳米粒子均匀分散在 TNTs 的顶端，而一些更小的粒子则负载在 TNTs 的内部。 此外, 他们还用类似的方法将 PtNi 纳米粒子负载在 $\mathrm{C}-\mathrm{TNTs}^{[35]}$ 的顶端和内部, 研究发现光照射能够明显 增强复合催化剂对甲醇氧化的催化活性。

利用电化学沉积法还可以合成不同形貌的贵金 属纳米结构。例如 Zhu 等 ${ }^{[27]}$ 以 TNTs 为载体, 先通 过电化学沉积法把 Pt 纳米花点缀在 TNTs 表面, 随 后又通过石墨烯修饰得到三元复合材料 $\mathrm{Pt}-\mathrm{TNTs} / \mathrm{RGO}$ 。如图 2 所示, 直径约 200 800 nm 的 $\mathrm{Pt}$ 纳米花分散在 TNTs 的顶部和侧面, 引入石墨烯 后, 纱状石墨烯覆盖在 Pt-TNTs 表面。Hu 等 ${ }^{[36]}$ 通过 恒电流沉积法把由多层 Pt 纳米片形成的纳米花沉 积在 TNTs 的表面。 $\mathrm{Du}$ 等 ${ }^{[37]}$ 以 $\mathrm{TiO}_{2} /$ 碳纤维 $(\mathrm{CF})$ 为 载体, 采用电化学沉积的方法先在 $\mathrm{TiO}_{2} / \mathrm{CF}$ 表面负 载具有三维(3D)结构的树枝状 $\mathrm{Ag}$ 纳米结构, 然后把 $\mathrm{Ag} / \mathrm{TiO}_{2} / \mathrm{CF}$ 电极作为工作电极浸入 $\mathrm{H}_{2} \mathrm{PtCl}_{6} / \mathrm{H}_{2} \mathrm{SO}_{4}$ 溶液中，通过置换反应得到了 $\mathrm{Ag} @ \mathrm{Pt}$ 双金属枝状结 构, 最后, 将片状石墨烯覆盖在 $\mathrm{Ag} @ \mathrm{Pt} / \mathrm{TiO}_{2} / \mathrm{CFs}$ 表 面形成复合电极。

\section{2 化学还原法}

化学还原法是通过贵金属前驱体与还原剂混 合, 然后在半导体表面沉积金属纳米结构得到贵金 属/半导体复合材料的方法 ${ }^{[41]}$ 。常用的还原剂有嗍氢 化钠 $\left(\mathrm{NaBH}_{4}\right)^{[32,38,42-48]}$ 、乙二醇 $(\mathrm{EG})^{[49-50]}$ 、油胺 $(\mathrm{OA})^{[28]}$ 和乙醇 ${ }^{[51-52]}$ 等。例如, Ramaraj 等 ${ }^{[42]}$ 用 $\mathrm{NaBH}_{4}$
还原纳米 $\mathrm{TiO}_{2}$ 和 $\mathrm{HAuCl}_{4}$ 混合溶液, 将 $\mathrm{Au}$ 纳米粒 子负载在多孔的 $\mathrm{TiO}_{2}$ 表面。 $\mathrm{Lu}$ 等 ${ }^{[50]}$ 把 $\mathrm{EG}$ 加入到 $\mathrm{H}_{2} \mathrm{PtCl}_{6}+\mathrm{NaOH}$ 的混合溶液中, 在 $\mathrm{N}_{2}$ 保护下 $160^{\circ} \mathrm{C}$ 回流 $3 \mathrm{~h}$, 将粒径约 $2 \mathrm{~nm}$ 的 $\mathrm{Pt}$ 纳米粒子均匀分散 在 $\mathrm{TiO}_{2} / \mathrm{C}$ 表面。Ravishankar 等 ${ }^{[28]}$ 以 $\mathrm{OA}$ 为还原剂, 采用原位合成法把 $\mathrm{Au}$ 纳米线修饰在 $\mathrm{ZnO}$ 纳米棒 表面。

\section{3 物理混合法}

对贵金属纳米粒子和半导体进行简单的物理混 合，也能够得到贵金属/半导体纳米复合物 ${ }^{[53-56]}$ 。例 如, Sugimoto 等 ${ }^{[53]}$ 首先通过注入法合成了 $\mathrm{C}$ 掺杂 $\mathrm{PtRu}$ 复合物, 然后在室温下将 $\mathrm{C}-\mathrm{PtRu}$ 粉末与 $\mathrm{TiO}_{2}$ 溶胶充分混合，最后在 $80^{\circ} \mathrm{C}$ 条件下烘干混合物，即 可得到所需产物。Polo 等 ${ }^{[55]}$ 将 $\mathrm{TiO}_{2} 、 \mathrm{Pt} 、 \mathrm{Ru}$ 和聚 合物前驱体混合在一起得到 $\mathrm{PtRu} / \mathrm{TiO}_{2} / \mathrm{C}$ 光电催化 剂。Shen 等 ${ }^{[57]}$ 用 $\alpha-\mathrm{Fe}_{2} \mathrm{O}_{3}$ 粉末与 $\mathrm{Pt}$ 前驱体混合后, 通过热处理得到 $\mathrm{Pt} / \alpha-\mathrm{Fe}_{2} \mathrm{O}_{3}$ 混合物。

\section{4 水热/溶剂热法}

水热/溶剂热法是合成纳米材料应用最广泛的技 术之一 ${ }^{[58], ~}$ 它的突出优点是反应设备简单, 粒子分 散性好、纯度高、晶形好且尺寸可控; 合成条件温和、 体系稳定, 且允许晶体在有毒体系中合成。与其他制 备方法相比，水热/溶剂热法更容易控制纳米材料的 取向和形态。例如, Hsu 课题组 ${ }^{[23]}$ 通过颈基丙氨酸辅 助水热法合成了独特的 $\mathrm{Au} / \mathrm{ZnS}$ 核壳结构, 其中壳的 厚度可以通过 $\mathrm{Au}$ 纳米粒子的浓度进行调节。Perng 等 ${ }^{[26]}$ 采用原子沉积技术和水热联合方法将直径为 $25 \mathrm{~nm}$ 的 $\mathrm{ZnO}$ 纳米棒负载在碳布上, 并进一步将 $\mathrm{Pt}$ 纳米粒子沉积在 $\mathrm{ZnO}$ 纳米棒表面。 $\mathrm{Lin}$ 课题组 ${ }^{[59]}$ 采 用水热法合成了 $\mathrm{Pt}(\mathrm{Pd}) / \mathrm{ZnO} /$ 石墨烯复合材料, 合成 的 $\mathrm{Pt}$ 和 $\mathrm{Pd}$ 纳米粒子的直径分别为 $3 \mathrm{~nm}$ 和 $5.5 \mathrm{~nm}$ 。 采用类似的方法, 他们又将直径为 $2 \mathrm{~nm}$ 的 Pt 纳米粒 子负载在 $\mathrm{SnO}_{2}$ /石墨烯表面形成了光电阳极 ${ }^{[60]}$ 。

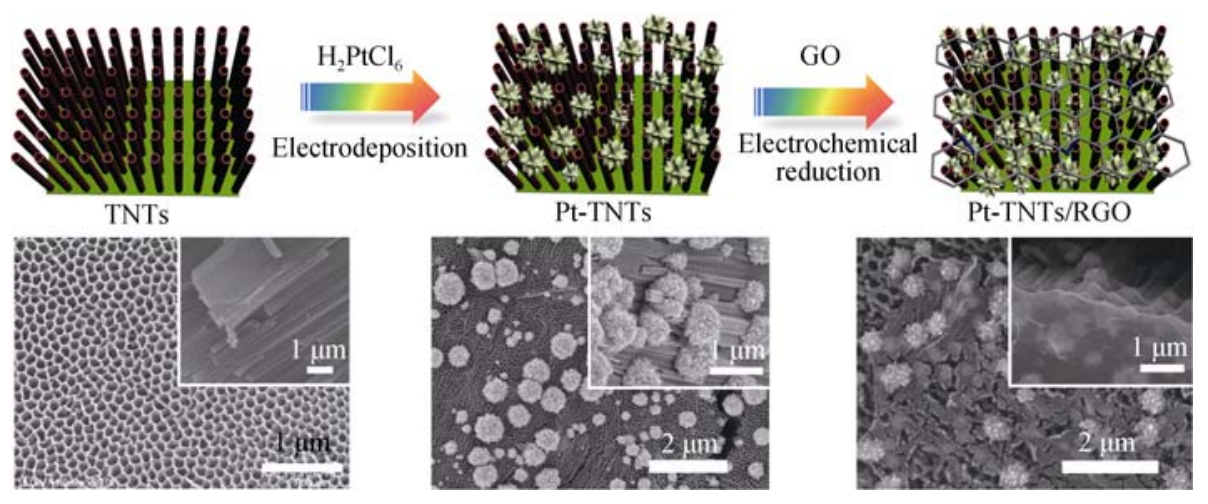

图 2 Pt-TNTs 和 Pt-TNTs/RGO 的制备和其 SEM 照片 ${ }^{[27]}$

Fig. 2 Schematic diagram and SEM images of Pt-TNTs and Pt-TNTs/RGO ${ }^{[27]}$ 


\section{5 微波辅助法}

微波辅助法是合成纳米材料的一种很有前途的 方法, 它的最突出优点是能够为整个样品提供一个 快速均匀的加热环境, 使之在很短时间内形成均匀 的纳米核。Lin 等 ${ }^{[61]}$ 以乙醇为还原剂, 采用微波辅助 法合成了 $\mathrm{Pt} / \mathrm{TiO}_{2}$ /石墨烯复合材料。在微波辅助下, $\mathrm{TiO}_{2}$ 不仅提高了乙醇的还原能力, 同时促进了 $\mathrm{Pt}$ 的 异相成核, 从而在 $\mathrm{TiO}_{2}$ 和石墨烯纳米片表面形成了 树枝状 Pt 纳米结构。此外, 他们还以葡萄糖为还原 剂, 在碱性环境中采用微波辅助法分别合成了 $\mathrm{Pt} / \mathrm{Cu}_{2} \mathrm{O}$ /石墨烯和 $\mathrm{Pd} / \mathrm{Cu}_{2} \mathrm{O}$ /石墨烯复合材料, 其中 $\mathrm{Cu}_{2} \mathrm{O}$ 在制备过程中能够提供分散点位以促进 $\mathrm{Pt}(0)$ 和 $\operatorname{Pd}(0)$ 的生长 ${ }^{[62]}$ 。

\section{6 其他方法}

除了上述方法, 研究人员还开发出多种合成方 法。例如 Lin 等 ${ }^{[63]}$ 采用简单的两步置换法合成了 Pt-Ag 树枝状纳米叶。Park 等 ${ }^{[64]}$ 采用溅射法成功将 直径为 $2 \mathrm{~nm}$ 的 $\mathrm{Pt}$ 纳米簇负载在 $\mathrm{TiO}_{2}$ 表面。Zhang 等 ${ }^{[65]}$ 在紫外光照射下将含有 $\mathrm{H}_{2} \mathrm{PtCl}_{6}$ 的溶液还原为 $\mathrm{Pt}$ 纳米粒子, 再通过物理混合的方法将其负载在用 亚甲基蓝改性的碳纳米管表面。

\section{3 光响应贵金属/半导体阳极催化剂}

贵金属/半导体作为新型阳极催化剂代替传统 贵金属催化剂, 在降低催化剂成本的同时能够提高 其催化性能。基于此, 本文总结了不同半导体纳米 材料与贵金属构成的复合催化剂在光照条件下增强 其催化性能的研究进展。

\section{$3.1 \mathrm{TiO}_{2}$ 纳米结构载体}

作为经典的光催化剂, $\mathrm{TiO}_{2}$ 及其复合材料被广 泛应用于环境和能源等领域 ${ }^{[18-19]}$ 。研究者利用 $\mathrm{TiO}_{2}$ 作为载体, 尝试与各种单金属纳米材料, 如 $\mathrm{Pt}^{[37,40,43,49-50,62-63]} 、 \mathrm{Au}^{[42,48,55]}$ 等或者多金属合金, 如 $\mathrm{PtRu}^{[21,52,54-55]} 、 \mathrm{PtNiRu}^{[39]}$ 等复合, 制备贵金属 $/ \mathrm{TiO}_{2}$ 复合电极。研究表明, 光照射可以有效提高复合电 极对甲醇的电催化氧化性能。例如, Kamat 课题组 ${ }^{[21]}$ 首次在紫外光照下将合成的 $\mathrm{Pt}-\mathrm{Ru} / \mathrm{TiO}_{2}$ 体系应用于 甲醇氧化并考察其催化机理, 研究发现 $\mathrm{Pt}-\mathrm{Ru}$ 和 $\mathrm{TiO}_{2}$ 均对甲醇有催化作用: $\mathrm{TiO}_{2}$ 的存在能够在增大 $\mathrm{Pt}-\mathrm{Ru}$ 催化剂表面积的同时增强其抗毒化性能; 在 紫外光照射下, $\mathrm{Pt}-\mathrm{Ru} / \mathrm{TiO}_{2}$ 的电流密度提高了 $25 \%$ 。该研究拓展了半导体催化剂的应用范围, 在 减少 DMFCs 中贵金属含量的同时提高了电池的 催化性能。
除 $\mathrm{Pt}$ 外, $\mathrm{Au}$ 纳米簇在甲醇电催化氧化中也起着 重要作用。KANG 等 ${ }^{[57]}$ 制备了商业 $\mathrm{TiO}_{2}$ 修饰的多 孔 $\mathrm{Au}$ 电极并考察其对甲醇的电/光电催化性能, 研 究发现, 在紫外光照射下复合催化剂对甲醇表现出 较大的光电流和几乎可逆的循环伏安响应, 说明 $\mathrm{TiO}_{2}$ 和 $\mathrm{Au}$ 之间存在的协同效应能够有效消除 $\mathrm{Au}$ 表面的钝化。

\section{2 一维 $\mathrm{TiO}_{2}$ 纳米管阵列(TNTs)}

最近研究发现, 以一维 TNTs 为贵金属载体形 成的复合催化剂对甲醇的电催化活性明显优于传统 的 $\mathrm{TiO}_{2}$ 纳米粒子 ${ }^{[66-67]}$ 。Xiao 课题组 ${ }^{[22,34-35]}$ 发现光照 条件下 TNTs 基催化剂的催化活性明显增强, 这主 要是因为电催化和光催化之间存在相互作用。 $\mathrm{He}$ 等 ${ }^{[32}$ 将 $\mathrm{Pt}$ 沉积在条纹状介孔 $\mathrm{TiO}_{2}$ 薄膜表面形成复 合材料, 因为光增强效应, 复合催化剂表现出更好 的电催化性能。在紫外光照射下, $\mathrm{Pt}$ 和 $\mathrm{TiO}_{2}$ 之间这 种独特的结构以及特殊的协同作用大大提高了催化 剂对甲醇氧化的电催化活性, 在开发新一代 DMFCs 中表现出很大的潜力。

另外, 研究还发现, $\mathrm{TiO}_{2}$ 基光电催化剂在醇类 氧化中不仅具有很高的催化活性, 而且具有自清洁 能力 ${ }^{[29,31,44-45]}$ 。Gao 等 ${ }^{[29]}$ 发现因为 TNTs 具有大的比 表面积、优秀的电子传输能力和光催化性能, 合成 的 $\mathrm{Pt} / \mathrm{TNTs}$ 复合催化剂不仅具有很高的催化活性, 而且具有很强的自清洁能力, 从而使催化剂能够循 环使用。经过紫外光处理, Pt/TNTs 电极对甲醇氧化 的催化活性几乎不变(图 3)。Hosseini 课题组 ${ }^{[31,44-46]}$ 做了一系列的研究工作发现, 由于 $\mathrm{TiO}_{2}$ 优异的光催 化性能, Pt/TNTs 复合电极经过短暂的紫外光处理即 可重复使用, 经过紫外光清洁的 Pt/TNTs 复合电极 对甲醇氧化性能几乎不变。

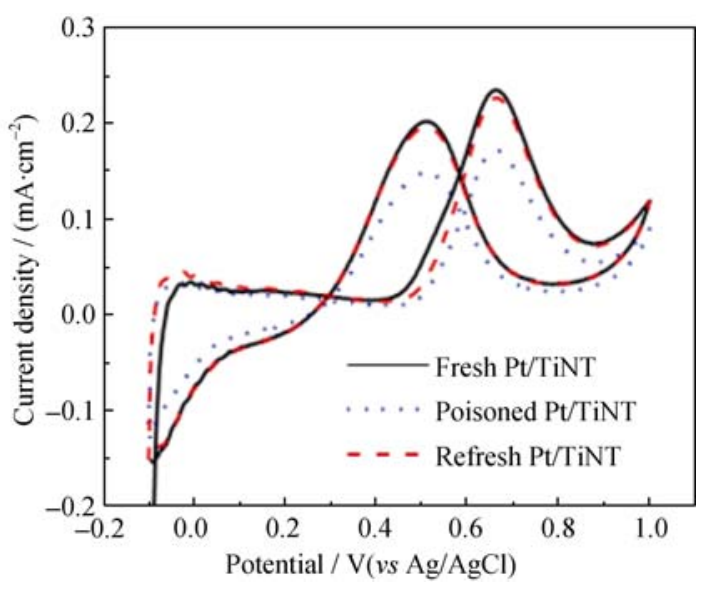

图 $3 \mathrm{Pt} / \mathrm{TiNT}$ 电极的自清洁曲线图 ${ }^{[29]}$

Fig. 3 Voltammetric response of the fresh, poisoned and refresh $\mathrm{Pt} /$ TiNT samples in a $1 \mathrm{~mol} / \mathrm{L} \mathrm{CH}_{3} \mathrm{OH}+1 \mathrm{~mol} / \mathrm{L} \mathrm{H}_{2} \mathrm{SO}_{4}$ solution ${ }^{[29]}$ 
然而，上述研究主要局限于紫外光照射，而紫 外光只占太阳光谱的 $5 \%$, 可见光约占 $45 \%{ }^{[20,68]}$ 。为 了高效地利用太阳能资源, 更多研究开发了基于可 见光响应的光催化材料。Zhu 等 ${ }^{[27]}$ 设计了一种对可 见光响应的光阳极, 该阳极是通过将 $\mathrm{Pt}$ 纳米花 /TNTs 与石墨烯复合得到的。研究发现, 利用石墨烯 与 TNTs 之间的相互作用, 电极在可见光下具有较 好的活性，使得复合电极对甲醇氧化的电催化活性 和稳定性在可见光照射下得到明显提高。该结果说 明可见光照射能够提高阳极催化剂对醇类氧化的催 化活性和稳定性。

\section{3 其他半导体载体}

此外, 其他半导体纳米材料如 $\mathrm{WO}_{3}-\mathrm{TiO}_{2}{ }^{[25,48]}$ 、 $\mathrm{ZnS}^{[23]} 、 \mathrm{ZnO}^{[26]}$ 和 $\mathrm{Cu}_{2} \mathrm{O}^{[63]}$ 等也被作为贵金属载体应 用于 DMFCs 中。如 Lin 等 ${ }^{[63]}$ 发现 $\mathrm{Pt} / \mathrm{Cu}_{2} \mathrm{O}$ /石墨烯 和 $\mathrm{Pd} / \mathrm{Cu}_{2} \mathrm{O}$ /石墨烯复合催化剂对甲醇的电催化活 性、稳定性和抗 $\mathrm{CO}$ 毒化能力均优于商业 $\mathrm{Pt} / \mathrm{C}$ 和商 业 $\mathrm{Pd} / \mathrm{C}$; 在紫外光照射下, $\mathrm{Pt} / \mathrm{Cu}_{2} \mathrm{O}$ / 石墨烯和 $\mathrm{Pd} / \mathrm{Cu}_{2} \mathrm{O}$ /石墨烯对甲醇的催化性和稳定性进一步增 强。 $\mathrm{He}$ 课题组 ${ }^{[25]}$ 以有序介孔 $\mathrm{WO}_{3}-\mathrm{TiO}_{2}$ 薄膜为载体 负载 $\mathrm{Pt}$ 催化剂并应用于 DMFCs。结果发现, $\mathrm{WO}_{3}$ 和 $\mathrm{TiO}_{2}$ 之间形成的异质结明显提高了复合半导体 表面电子-空穴分离效率。另外, 在紫外光照射下, 由于 $\mathrm{Pt}$ 和空穴之间存在特殊的协同效应, $\mathrm{Pt} / \mathrm{TiO}_{2}-\mathrm{WO}_{3}$ 复合电极对甲醇氧化表现出优异的电 催化活性。该结果为设计新一代 DMFCs 提供了积 极的参考价值。

除了传统的半导体, 表面等离子体催化剂因其 独特的光学性能和稳定性, 也引起了研究人员的极 大兴趣 ${ }^{[69-72]}$ 。Tseng 等 ${ }^{[72]}$ 合成了具有三维结构树枝 状的纳米金并作为光电催化剂应用于甲醇氧化, 结 果发现可见光照射下纳米 $\mathrm{Au}$ 对甲醇的催化性能明 显增强。该研究极大地拓展了光响应阳极催化剂在 DMFCs 中的应用。

\section{4 总结和展望}

本文概述了近年来光响应贵金属/半导体电极 的制备方法、半导体载体类型及发展现状。以新型 半导体材料作为载体不仅能够降低贵金属的负载量, 还可以改善贵金属催化剂的催化活性和稳定性。尽 管半导体材料在电子传导性方面与传统贵金属或者 碳载体相比还需提高, 但是贵金属/半导体光电极具 有独特的光学和电学性能, 光照条件下它对甲醇的 催化具有光催化和电催化的协同效应, 且半导体材
料在光照作用下，其产生的空穴强氧化性使得该电 极具有明显的自清洁能力。与传统的贵金属阳极电 催化剂相比, 光照条件下这种独特的复合电极中贵 金属与半导体之间存在的协同效应能够大大促进甲 醇催化氧化, 在设计新一代 DMFCs 过程中表现出 巨大的潜力。根据已有的理论分析和研究总结, 我 们对光响应直接甲醇燃料电池的设备进行设想：以 贵金属/半导体为电池阳极, 采用透光材料封装电池, 电池运行时可以通过太阳光照射来提高阳极的甲醇 催化氧化性能; 电池阴极采用传统材料组装, 实现 氧气的还原。整体来说, 光响应直接甲醇燃料电池 可以把太阳能和甲醇内储化学能同时转化为电能。

总之, 光响应贵金属/半导体电极在 DMFCs 中 应用的研究已经取得了一定的进展, 其下一步研究 可以从以下几个方面出发: (1)合成方法的发展和创 新。该方面的重点在于研发绿色无污染，简单可控， 低成本和大规模的制备方法。(2)催化剂载体的选 择。该方面的重点在于探寻合适的半导体及其复合 材料来提高贵金属/半导体电极在光(特别是可见光) 照射下其对甲醇的催化性和稳定性。(3)光电催化机 理的深入探究。该方面的重点在于借助有效的表征 手段来研究光电催化过程中电子迁移和电荷转移的 情况。总之，随着科技的进步，我们将有机会设计更 有效的光响应贵金属/半导体复合电极并应用于 DMFCs，从而为实现新一代燃料电池大规模应用奠 定坚实的基础。

\section{参考文献:}

[1] ANDÚJAR J M, SEGURA F. Fuel cells: History and updating. A walk along two centuries. Energy Reviews, 2009, 13: 2309-2322.

[2] SHARMA S, POLLET B G. Support materials for PEMFC and DMFC electrocatalysts - a review. J. Power Sources, 2012, 208(15): 96-119.

[3] CAO M N, WU D S, CAO R. Recent advances in the stabilization of platinum electrocatalysts for fuel-cell reactions. ChemCatChem, 2014, 6: 26-45.

[4] ARICÒ A S, SRINIVASAN S, ANTONUCCI V. DMFCs: from fundamental aspects to technology development. Fuel Cells, 2001, 1: 133-161.

[5] WANG, Z H, SHI G Y, XIA J F, et al. Research progress on Pt-based anode catalysts in the direct methanol fuel cell. Acta Chim. Sinica, 2013, 71: 1225-1238.

[6] YU X W, PICKUP P G. Recent advances in direct formic acid fuel cells (DFAFC). J. Power Sources, 2008, 182: 124-132.

[7] LIN L, ZHU Q, XU A W. Anode catalysts and cathode catalysts of direct methanol fuel cells. Prog. Chem., 2015, 27(9): 1147-1157.

[8] KAKATI N, MAITI J, LEE S H, et al. Anode catalysts for direct methanol fuel cells in acidic media: Do we have any alternative for Pt or Pt-Ru? Chem. Rev., 2014, 114: 12397-12429.

[9] KOENIGSMANN C, WONG S S. One-dimensional noble metal electrocatalysts: A promising structural paradigm for direct methanol fuel cells. Eng. \& Environ. Sci., 2011, 4: 1161-1176. 
[10] ZHAO X, YIN M, MA L, et al. Recent advances in catalysts for direct methanol fuel cells. Eng. Environ. Sci., 2011, 4: 2736-2753.

[11] HUANG H J, WANG X J. Recent progress on carbon-based support materials for electrocatalysts of direct methanol fuel cells. Mater. Chem., 2014, 2: 6266-6291.

[12] ANTOLINI E. Composite materials: An emerging class of fuel cell catalyst supports. Appl. Catal. B: Environ., 2010, 100: 413-426.

[13] SHRESTHA S, LIU Y, MUSTAIN W E. Electrocatalytic activity and stability of Pt clusters on state-of-the-art supports: A review. Catal. Rev., 2011, 53: 256-336.

[14] LIU Y, GOKCEN D, BERTOCCI U, et al. Self-terminating growth of platinum films by electrochemical deposition. Science, 2012, 338(6112): 1327-1330.

[15] LV Q, YIN M, ZHAO X, et al. Promotion effect of $\mathrm{TiO}_{2}$ on catalytic activity and stability of Pt catalyst for electrooxidation of methanol. J. Power Sources, 2012, 218(12): 93-99.

[16] MURAWSKA M, COX J A, MIECZNIKOWSKI K. PtIr-WO nanostructured alloy for electrocatalytic oxidation of ethylene glycol and ethanol. J. Solid State Electrochem., 2014, 18(11): 3003-3010.

[17] ZHANG H, HU C, HE X, et al. Pt support of multidimensional active sites and radial channels formed by $\mathrm{SnO}_{2}$, flower-like crystals for methanol and ethanol oxidation. J. Power Sources, 2011, 196: $4499-4505$.

[18] LinSEBIGleR A L, LU G, YATES J T. Photocatalysis on $\mathrm{TiO}_{2}$ surfaces: principles, mechanisms, and selected results. Chem. Rev., 1995, 95(3): 735-758.

[19] SCHNEIDER J, MATSUOKA M, TAKEUCHI M, et al. Understanding $\mathrm{TiO}_{2}$ photocatalysis: mechanisms and materials. Chem. Rev., 2014, 114(19): 9919-9986.

[20] ZHOU H L, QU Y Q, ZEID T, et al. Towards highly efficient photocatalysts using semiconductor nanoarchitectures. Eng. Environ. Sci., 2012, 5(5): 6732-6743.

[21] DREW K, GIRISHKUMAR G, VINODGOPAL K, et al. Boosting fuel cell performance with a semiconductor photocatalyst: $\mathrm{TiO}_{2} / \mathrm{Pt}$-Ru hybrid catalyst for methanol oxidation. J. Phys. Chem. B, 2005, 109(24): 11851-11857.

[22] HE H. C, XIAO P, ZHOU M, et al. Boosting catalytic activity with a p-n junction: $\mathrm{Ni} / \mathrm{TiO}_{2}$, nanotube arrays composite catalyst for methanol oxidation. Int. J. Hydrogen Energy, 2012, 37(6): 4967-4973.

[23] CHEN W T, LIN Y K, YANG T T, et al. Au/ZnS core/shell nanocrystals as an efficient anode photocatalyst in direct methanol fuel cells. J. Chem. Comm., 2013, 49(76): 8486-8488.

[24] MOJUMDER N, SARKER S, ABBAS S A, et al. Photoassisted enhancement of the electrocatalytic oxidation of formic acid on platinized $\mathrm{TiO}_{2}$ nanotubes. ACS Appl. Mater. Interfaces., 2014, 6(8): 5585-5594.

[25] WANG T, TANG J, WU S C, et al. Preparation of ordered mesoporous $\mathrm{WO}_{3}-\mathrm{TiO}_{2}$, films and their performance as functional $\mathrm{Pt}$ supports for synergistic photo-electrocatalytic methanol oxidation. J. Power Sources, 2014, 248(7): 510-516.

[26] SU C Y, HSUEH Y C, KEI C C, et al. Fabrication of high-activity hybrid Pt@ZnO catalyst on carbon cloth by atomic layer deposition for photoassisted electro-oxidation of methanol. J. Phys. Chem. C, 2013, 117(22): 11610-11618.

[27] ZHAI C Y, ZHU M S, BIN D, et al. Visible-light-assisted electrocatalytic oxidation of methanol using reduced graphene oxide modified Pt nanoflowers- $\mathrm{TiO}_{2}$ nanotube arrays. ACS Appl. Mater. Inter., 2014, 6(20): 17753-17761.

[28] LEELAVATHI A, MADRAS G, RAVISHANKAR N. New insights into electronic and geometric effects in the enhanced photoelectrooxidation of ethanol using $\mathrm{ZnO}$ nanorod/ultrathin $\mathrm{Au}$ nanowire hybrids. J. Am. Chem. Soc., 2014, 136(41): 14445-14455.

[29] SONG Y Y, GAO Z D, SCHMUKI P. Highly uniform Pt nanoparticle decoration on $\mathrm{TiO}_{2}$, nanotube arrays: a refreshable platform for methanol electrooxidation. Electrochem. Commun., 2011, 13(3): 290-293.

[30] ZHAI C Y, ZHU M S, PANG F Z, et al. High efficiency photoelectrocatalytic methanol oxidation on CdS quantum dots sensitized $\mathrm{Pt}$ electrode. ACS Appl. Mater. Inter., 2016, 8: 5972-5980.

[31] HOSSEINI M G, MOMENI M M. UV-cleaning properties of Pt nanoparticle-decorated titania nanotubes in the electro-oxidation of methanol: an anti-poisoning and refreshable electrode. Electrochim. Acta, 2012, 70(6): 1-9.

[32] WU S C, HE J P, ZHOU J H, et al. Fabrication of unique stripe-shaped mesoporous $\mathrm{TiO}_{2}$ films and their performance as a novel photo-assisted catalyst support for DMFCs. J. Mater. Chem., 2011, 21(9): 2852-2854.

[33] FAN X L, ZHANG C X, XUE H R, et al. Fabrication of $\mathrm{SiO}_{2}$ incorporated ordered mesoporous $\mathrm{TiO}_{2}$ composite films as functional Pt supports for photo-electrocatalytic methanol oxidation. RSC Adv., 2015, 5(96): 78880-78888.

[34] HE H C, XIAO P, ZHOU M, et al. Preparation of well-distributed $\mathrm{Pt}-\mathrm{Ni}$ nanoparticles on/into $\mathrm{TiO}_{2}$ NTs by pulse electrodeposition for methanol photoelectro-oxidation. Catal. Commun., 2011, 16(1): 140-143.

[35] He H C, XIAO P, ZHOU M, et al. PtNi alloy nanoparticles supported on carbon-doped $\mathrm{TiO}_{2}$, nanotube arrays for photo-assisted methanol oxidation. Electrochim. Acta, 2013, 88(2): 782-789.

[36] LIU J, LIU B, NI Z Y, et al. Improved catalytic performance of $\mathrm{Pt} / \mathrm{TiO}_{2}$, nanotubes electrode for ammonia oxidation under UV-light illumination. Electrochim. Acta, 2014, 150: 146-150.

[37] WANG C Q, YUE R R, WANG H W, et al. Dendritic Ag@Pt core-shell catalyst modified with reduced graphene oxide and titanium dioxide: Fabrication, characterization, and its photo-electrocatalytic performance. Int. J. Hydrogen Energy, 2014, 39(11): 5764-5771.

[38] ROKESH K, PANDIKUMAR A, MOHAN S C, et al. Aminosilicate Sol-Gel supported zinc oxide-silver nanocomposite material for photoelectrocatalytic oxidation of methanol. J. Alloys Compd., 2016, 680: 633-641.

[39] CHU D B, WANG S X, ZHENG P, et al. Anode catalysts for direct ethanol fuel cells utilizing directly solar light illumination. ChemSusChem, 2009, 2(2): 171-176.

[40] WANG C Q, JIANG F X, YUE R R, et al. Enhanced photo-electrocatalytic performance of $\mathrm{Pt} / \mathrm{RGO} / \mathrm{TiO}_{2}$ on carbon fiber towards methanol oxidation in alkaline media. J. Solid State Electrochem., 2014, 18(2): 515-522.

[41] SANKAR M, DIMITRATOS N, MIEDZIAK P J, et al. Designing bimetallic catalysts for a green and sustainable future. Chem. Soc. Rev., 2013, 41(12): 8099-8139.

[42] PANDIKUMAR A, MURUGESAN S, RAMARAJ R. Functionalized silicate Sol-Gel-supported $\mathrm{TiO}_{2}$-Au core-shell nanomaterials and their photoelectrocatalytic activity. ACS Appl. Mater. Inter., 2010, 2(7): 1912-1917.

[43] ZHANG H M, ZHOU W Q, DU Y K, et al. Enhanced electrocatalytic performance for methanol oxidation on $\mathrm{Pt}_{-} \mathrm{TiO}_{2} / \mathrm{ITO}$ electrode under UV illumination. Int. J. Hydrogen Energy, 2010, 35(24): 13290-13297.

[44] HOSSEINI M G, MOMENI M M. Platinum nanoparticle-decorated $\mathrm{TiO}_{2}$, nanotube arrays as new highly active and non-poisoning catalyst for photo-electrochemical oxidation of galactose. Appl. Catal. A: Gen., 2012, 427-428(10): 35-42.

[45] HOSSEINI M G, MOMENI M M. Evaluation of the performance of platinum nanoparticle-titanium oxide nanotubes as a new re- 
freshable electrode for formic acid electro-oxidation. Fuel Cells, 2012, 12(3): 406-414.

[46] HOSSEINI M G, MOMENI M M. Fabrication and photoelectrocatalytic activity of highly oriented titania nanotube loaded with platinum nanoparticles for electro-oxidation of lactose: a new recyclable electro-catalyst. J. Mol. Catal. A: Chem., 2012, 355: 216-222.

[47] PANDIYARAJAN C, PANDIKUMAR A, RAMARAJ R. Photoelectrocatalytic performance of a titania-keggin type polyoxometalate-gold nanocomposite modified electrode in methanol oxidation. Nanotechnology, 2013, 24(43): 435401-435408.

[48] WANG C Q, JIANG F X, RONG Z, et al. Enhancement of methanol electrocatalytic oxidation on platinized $\mathrm{WO}_{3}-\mathrm{TiO}_{2}$ composite electrode under visible light irradiation. Mater. Res. Bull., 2013, 48(3): 1099-1104.

[49] LIN C T, HUANG H J, YANG J J, et al. A simple fabrication process of $\mathrm{Pt}-\mathrm{TiO}_{2}$ hybrid electrode for photo-assisted methanol fuel cells. Microelectron. Eng., 2011, 88(8): 2644-2646.

[50] LI W, BAI Y, LI F J, et al. Core-shell $\mathrm{TiO}_{2} / \mathrm{C}$ nanofibers as supports for electrocatalytic and synergistic photoelectrocatalytic oxidation of methanol. J. Mater. Chem., 2012, 22: 4025-4031.

[51] HSU Y H, NGUYEN A T, CHIU Y H, et al. Au-decorated GaOOH nanorods enhanced the performance of direct methanol fuel cells under light illumination. Appli. Catal. B: Environ., 2015, 185: 133-140.

[52] XIE J, ZHANG Q H, GU L, et al. Ruthenium-platinum core-shell nanocatalysts with substantially enhanced activity and durability towards methanol oxidation. Nano Energy, 2016, 21: 247-257.

[53] SAIDA T, OGIWARA N, TAKASU Y, et al. Titanium oxide nanosheet modified PtRu/C electrocatalyst for direct methanol fuel cell anodes. J. Phys. Chem., 2010, 114(31): 13390-13396.

[54] ARULMani D V, EASTCOTT J I, MAVILla S G, et al. Photoenhanced activity of Pt and Pt-Ru catalysts towards the electrooxidation of methanol. J. Power Sources, 2014, 247(2): 890-895

[55] POLO A S, SANTOS M C, SOUZA R F B, et al. Pt-Ru-TiO 2 photoelectrocatalysts for methanol oxidation. J. Power Sources, 2011, 196: $872-876$.

[56] JIA C C, YIN H M, MA H, et al. Enhanced photoelectrocatalytic activity of methanol oxidation on $\mathrm{TiO}_{2}$-decorated nanoporous gold. J. Phys. Chem. C, 2009, 113(36): 16138-16143.

[57] KANG S, SHEN P K. Facial synthesis of porous hematite supported Pt catalyst and its photo enhanced electrocatalytic ethanol oxidation performance. Electrochim. Acta, 2015, 168(10): 104-110.

[58] SHI W D, SONG S Y, ZHANG H J. Hydrothermal synthetic strategies of inorganic semiconducting nanostructures. Chem. Soc. Rev., 2013, 42(13): 5714-5743.
[59] LI Z S, YE L T, LEI F L, et al. Enhanced electro-photo synergistic catalysis of $\mathrm{Pt}(\mathrm{Pd}) / \mathrm{ZnO} /$ graphene composite for methanol oxidation under visible light irradiation. Electrochim. Acta, 2015, 188: 450-460.

[60] LEI F L, LI Z S, YE L T, et al. One-pot synthesis of $\mathrm{Pt} / \mathrm{SnO}_{2} / \mathrm{GNs}$ and its electro-photo-synergistic catalysis for methanol oxidation. Int. J. Hydrogen Energy, 2016, 41(1): 255-264.

[61] YE L T, LI Z S, ZHANG L, et al. A green one-pot synthesis of $\mathrm{Pt} / \mathrm{TiO}_{2} /$ graphene composites and its electro-photo-synergistic catalytic properties for methanol oxidation. J. Colloid Inter. Sci., 2014, 433(11): 156-162.

[62] YE L T, LI Z S, ZHANG L, et al. One-step microwave synthesis of $\mathrm{Pt}(\mathrm{Pd}) / \mathrm{Cu}_{2} \mathrm{O} / \mathrm{GNs}$ composites and their electro-photo-synergistic catalytic properties for methanol oxidation. J. Mater. Chem. A, 2014, 2(48): 21010-21019.

[63] LIN C T, SHIAO M H, CHANG M N, et al. A facile approach to prepare silicon-based Pt-Ag tubular dendritic nano-forests (tDNFs) for solar-light-enhanced methanol oxidation reaction. Res. Lett., 2015, 10(1): $1-8$.

[64] PARK K W, HAN S B, LEE J M. Photo(UV)-enhanced performance of Pt- $\mathrm{TiO}_{2}$, nanostructure electrode for methanol oxidation. Electrochem. Commun., 2007, 9(7): 1578-1581.

[65] XU M Li, DUAN BEN, ZHANG Y J, et al. Effect of modification factors of MWCNTs support on electrocatalytic performance of $\mathrm{Pt}$ nanoparticles. J. Inorg. Mater., 2015, 30(9): 931-936.

[66] SONG H Q, QIU X P, LI X X, et al. $\mathrm{TiO}_{2}$, nanotubes promoting $\mathrm{Pt} / \mathrm{C}$ catalysts for ethanol electro-oxidation in acidic media. $J$. Power Sources, 2007, 170(1): 50-54.

[67] XING L, JIA J B, WANG Y Z, et al. Pt modified $\mathrm{TiO}_{2}$ nanotubes electrode: Preparation and electrocatalytic application for methanol oxidation. Int. J. Hydrogen Energy, 2010, 35(22): 12169-12173.

[68] ZHU M S, CHEN P L, LIU M H. Ag/AgBr/Graphene oxide nanocomposite synthesized via oil/water and water/oil microemulsions: A comparison of sunlight energized plasmonic photocatalytic activity. Langmuir, 2012, 28(7): 3385-3390.

[69] ZHANG X M, CHEN Y L, LIU R S, et al. Plasmonic photocatalysis. Rep. Prog. Phys., 2013, 76(4): 2020-2027.

[70] ZHU M S, CHEN P L, LIU M H. Ag/AgX(X = CI, Br, I): A new type plasmonic photocatalysts. Prog. Chem., 2013, 25(2): 209-220.

[71] ZHU M S, CHEN P L, LIU M H. Graphene oxide enwrapped $\mathrm{Ag} / \mathrm{AgX}(\mathrm{X}=\mathrm{Br}, \mathrm{Cl})$ nanocomposite as a highly efficient visible-light plasmonic photocatalyst. ACS Nano, 2011, 5(6): 4529-4536.

[72] LIN C T, CHANG M N, HUANG H J, et al. Rapid fabrication of three-dimensional gold dendritic nanoforests for visible light-enhanced methanol oxidation. Electrochim. Acta, 2016, 192: $15-21$. 